\title{
A new approach for extending the possibilities of collaboration between BIM, GIS and Web environments to increase the efficiency of building space management
}

\author{
Danylo Shkundalov ${ }^{1}$, Tatjana Vilutiené ${ }^{2}$ \\ Department of Construction Management and Real Estate, Vilnius Gediminas Technical University, \\ Vilnius, Lithuania \\ E-mail: 'daniil.shkundalov@null.net (corresponding author)
}

\begin{abstract}
The paper presents the approach that extends the opportunities of Web environments in BIM model processing and allows implementation of the bigger amount of analysis that is based on the object's positioning. With the developed relations, the objects in the BIM model can be considered not just like independent 3D objects in the WebGL environment but as objects that are linked between each other and can act by the rules that defined to each type of the objects. Correlated co-ordinates will be useful for the architects, building operators and facility managers who use the BIM model for the management of the building and its components. This method can be used to increase the effectiveness of space management, clash detection, relocation management and space programming as well as existing conditions modelling and lighting analysis can be done in a way that is more efficient due to possibility of implementation of the survey coordinate system that will give the coordinates to each object in the scene.
\end{abstract}

Keywords: building space management, efficiency, BIM, GIS, Web.

\section{Introduction}

Nowadays, people spend the majority of their time inside of buildings. Due to fast technological advancements, buildings are becoming more complex and sophisticated. As complex sets of interdependent components, materials, machinery, infrastructure and people, with many spatial relationships and dependencies; buildings require more sophisticated tools for design and management. GIS tools have been used successfully in a broad range of applications inside and outside of buildings, such as environmental analysis and landscape planning, operations planning, safety and security planning, space utilization and optimization. Geographic information systems (GIS) designed specifically for the analysis of spatial relationships and therefore, offer many benefits to the facilities management (Dangermond, 2010).

Web Graphics Library or WebGL is a JavaScript API that allows web browsers to render interactive 3D and 2D graphics due to OpenGL ES 2.0 that can be used in HTML5 canvas elements. This technology can be used to visualize and process BIM models in web browsers, however, this process faces a big amount of problems. The main issues related to the difference of the coordinate systems in these two environments and the ways in which coordinates are calculated. Every object presented in the WebGL environment needs to be placed inside the scene. The scene is a 3D space defined by the WebGL local coordinate system that is used to place all types of objects relative to the centre coordinate system. The coordinate system of a scene allows visualization of the 3D objects in their places and manipulates with them, however, it cannot be used to process BIM model objects properly, because object positioning in BIM environment has a significant difference compared to the WebGL environment. There is a big number of projects, which provide the opportunity to visualize the BIM models in the Web environment. However, most of them allow just visualization and no manipulation due to issues that are related to this process. Most of the Web based solutions that provide tools for manipulating with the objects solves these issues by dividing the separate 3D objects into groups, but such solution cannot be used for implementation of the analysis due to deficiency of the objects' parameters and their relations, as they are usual 3D objects with extra information. This situation appears because objects in a Web environment contain geometric information of the object that includes shape, texture and coordinates to the centre of the coordinate system. These parameters are sufficient for processing of a 3D object in WebGL environment, however, the BIM model needs more sophisticated information and cannot be properly processed by this set of parameters. Every

(C) 2019 Authors. Published by VGTU Press. This is an open-access article distributed under the terms of the Creative Commons Attribution (http://creativecommons.org/licenses/by/4.0/) License, which permits unrestricted use, distribution, and reproduction in any medium, provided the original author and source are credited. 
object in the BIM model contains geometric and attributive information about this object and relations with other objects. These relations are necessary for the positioning, processing and analysis of BIM model objects in a BIM environment. WebGL environment can not process native BIM file formats, therefore, they need to be converted into supportable file formats such as IFC, JSON, JS, etc. The conversion process cannot derive all parameters from the BIM model into a resulting file that means that resulting file will be presented as a usual 3D object with extra information.

The paper presents the approach that extends the opportunities of BIM model processing in a Web environment by adding lost parameters and dependencies. The latter allows deeper analysis that is based on the object's positioning and parameters. This approach is based on the reworking of the BIM model and WebGL handling logic and consists of several steps.

\section{Literature review}

Integration between Building Information Models (BIM) and Geographic Information Systems (GIS) has been a major problem that practitioners of the field recently face. Many conceptual models designed for visualization purposes (Li \& He, 2008; Irizarry, Karan, \& Jalaei, 2013; Borrmann et al., 2015; Niu, Pan, \& Zhao, 2015). However, semantic models are more needed for different engineering and planning applications to enable complex queries and analysis (Hor, Jadidi, \& Sohn, 2016). The purpose of this integration is to meet the increased demand for building systems analysis, urban planning applications, cadastre and security and many other applications (Hor, 2015). These applications do not only require 3D geometry and appearance information, they require complex semantic information. Many researchers from both GIS and BIM communities have been working on developing conceptual models in forms of geometric and semantic models, mostly for efficient representation of 3D objects. For example, in order to integrate heterogeneous data, such as BIM, GIS, and FM data, Kang and Hong (2015) proposed a method to map FM data from BIM to GIS by using a BIM/GIS-based information Extract, Transform, and Load (BG-ETL) method. Hor et al. (2016) proposed a novel approach for integrating BIM and GIS using semantic web technologies and Resources Description Framework (RDF) graphs.

The integration of BIM and geospatial analyses can offer substantial benefits to manage the planning process during the design and pre-construction stages. However, this integration suffers from a lack of interoperability across the geospatial and BIM domains. Karan and Irizarry (2015) proposed the interoperability mechanism by employing semantic web technology. The proposed approach consists of translation of the building's elements and GIS data into a semantic web data format, the use of a set of standardized ontologies for construction operations to integrate and query the heterogeneous spatial and temporal data, and the use of a query language to access and acquire the data in semantic web format. Kim, Shin, Choe, Seibert, and Walz (2012) proposed a prototype of an energy monitoring system called EnerGIS that is Web-based rich-internet application (RIA) integrating a 3D geospatial viewer based on the Google Earth platform and Google Maps components with additional data visualization modules.

Despite many attempts in the integration of BIM and geospatial analyses, future studies could further enhance the interoperability of GIS and BIM-based information. Wong, Ge, and He (2018) pointed out that interoperability of different data forms, including spatial data, temporal data and informational data is still an unsolved issue. The review made by Liu et al. (2017) revealed that there are still many obstacles and challenges to the achievement of BIM and GIS integration. Mismatching information between these two environments is one of the most important factors. The mismatching information not only indicates the standard format difference, but also stands for other differences such as different users, application focuses, developmental stages, spatial scales, coordinate system, semantic and geometric representations, levels of granularity, and different information storage and access methods. The review made by Pärn, Edwards, and Sing (2017) revealed that data within BIM for FM is not fully exploited for decision support. Therefore, the opportunity to enhance a building's performance using rich semantic data is lost. This issue further worsened by gaps in software interoperability when transitioning between as-built BIM and a CAFM system. Subsequently, the broad range of geometric and semantic data embedded in the BIM model points to the potential to augment data analysis and generate accurate knowledge capture and decision-making.

The analysis of recent developments made by Pauwels, Zhang, and Lee (2017) revealed that no approach for defining semantics and sharing requirements exists; exchange specifications have been separately defined and executed in different ways, which results in a lack of consistency. Thus, as an effort to formalize exchange specifications and organize them in a well-structured classification, semantic web technologies could be employed for defining and sharing IDM and MVD requirements. For future research, Ma and Ren (2017) suggest researchers focus on issues including the use of a unified model, based on the popular standard data format of IFC and CityGML on multiple purposes across the whole building lifecycle. The review made by Zhu, Wright, J. Wang, and X. Wang (2018) revealed new issues that may arise. One that could be envisioned is the impact of huge data handling. New techniques are needed to handle this issue, such as innovative methods for reducing data size while keeping semantic information intact. Another challenge might be developing efficient methods for model creation. The current process for creating city models is still cumbersome and time-consuming, especially for those with a high level of detail. New efficient methods are needed to facilitate this process for a city model to be built quickly and efficiently. 


\section{Proposed model for collaboration between BIM, GIS and Web environments}

The proposed approach is based on the reworking of the BIM model and WebGL handling logic and consists of the following steps:

Step 1. Determination of the in-model relations.

Step 2. Calculation of the correlated coordinates for the objects.

Step 3. Creation of the relations of the objects.

Step 4. Recalculation of the objects coordinates in the WebGL environment coordinate system.

Step 5. The rebuilding of the BIM model based on new coordinates.

\section{Step 1. Determination of the in-model relations.}

The determination of the relations inside the model is a primary and important step that can be done by using attributive information of the elements inside the BIM model. The main part of this process is to find the host object, the parameters of which will be inherited by its child objects. In a converted BIM model, every object contains some attributive field with the information about related host element.

In the example presented in Figure 1 the host element for the Object3 is Object2, and host element for the Object2 is Objectl that means that relation sequence for the Object 3 will be Object 2 and Object1. When the sequence is determined, the child object will be linked to the host object and receive full relation sequence that will be used for manipulations and analysis. The sequence of relations should be set for all objects in the model.

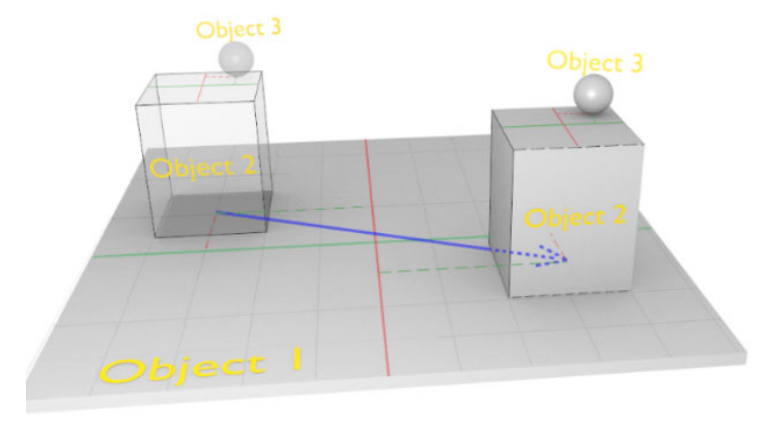

Figure 1. Relations of the objects

\section{Step 2. Calculation of the correlated coordinates for the objects.}

Every object in the WebGL scene environment is placed respectfully to the centre of the scene coordinate system that is used to visualize model by default, however, these coordinates cannot be used for analysis because they do not represent the positions of the objects related to each other. The defined relations give the opportunity to calculate the correlated coordinates of the objects whereby the position of the objects can be determined. That is highly important for space management and some analysis such as clash detection. In this step, scene coordinates should be recalculated respectfully to the host elements. In the example presented in Figure 1, the central point of the Object1 needs to be defined as dependable from the parameters such as width and height. Object's coordinates correlated to the host object can be calculated using the formula (1), where $x_{n}$ and $y_{n}$ - coordinates of the object's center; $x_{n-1}$ and $y_{n-1}-$ coordinates of the host object's center; $h_{n}$ and $h_{n-1}$ - height of the object and host object respectfully. These coordinates will be used not just to manipulate with the object in the response of host object manipulation, but also to calculate geodesic coordinates in case they were provided. The sum of the correlated coordinates can be checked by comparison with scene native coordinates - they need to be the same in the moment of model reconstruction, however, all future manipulations with the objects will be done by use of the correlated coordinates.

$$
x_{n}^{C} y_{n}^{C}=\left[\left(\begin{array}{c}
\left(x_{n}-x_{n-1}\right) \\
\left.y_{n}-\frac{h_{n}}{2}-y_{n-1}+\frac{h_{n-1}}{2}\right)
\end{array}\right] .\right.
$$

\section{Step 3. Creation of the relations of the objects.}

After the correlated coordinates are calculated, the dependencies of the objects need to be developed with the purpose of tracking the changes of the host objects and change child coordinates in response - this conception can be granted due to JavaScript technology. In this step, the rules for the objects need to be implemented. The type of the rules are determined by the object's type, for example, window cannot be placed outside of the wall and the wall need to be constructed on the top of the floor. Moreover, rules need to fit the parameters of the object and WebGL object presentation need to be changed in case it is necessary. For example, window's glass has transparency and heat flow 
parameters, which means that glass needs to have opacity in WebGL environment visualisation and heat flow have to be calculated in solar analysis. The rules are going to be used every time model changed or analyzed. The pseudofunction responsible for tracking such manipulations is presented in Figure 2, where coordinates for each child objects are calculated via recursion, and check for the rules is presented in the CheckForRules pseudo-function that depicts an error in case of issues and stops movement of objects.

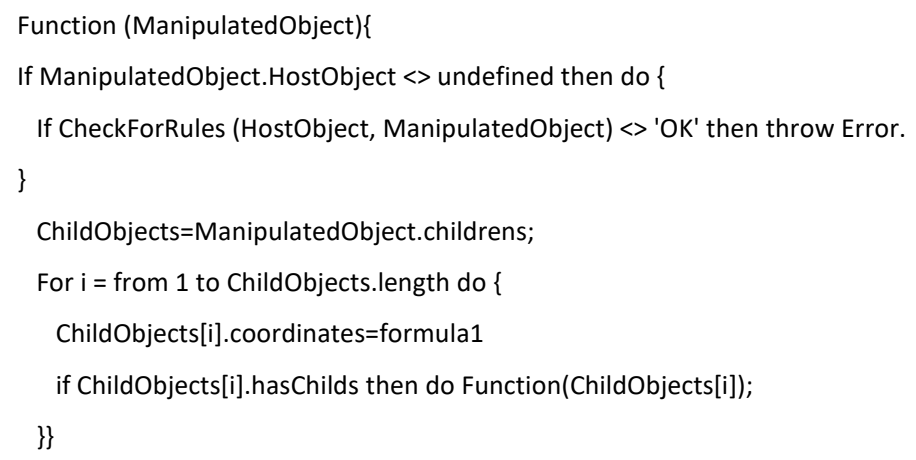

Figure 2. Object manipulation-tracking function

Step 4. Recalculation of the objects coordinates in the WebGL environment coordinate system.

WebGL environment cannot visualize objects of the model by use of correlated coordinates, thus scene coordinates need to be calculated by the relation sequence. The sequence represents the sum of the coordinates for each object in the model determined from every host object. Formula (2) presents the calculation of the scene coordinates of the objects based on the sequence of hosts objects, where $x_{i}^{C}$ and $y_{i}^{C}$-correlated coordinates for the object in the sequence, and $h_{n}$ is a height of the object.

$$
y_{n}^{S} x_{n}^{S}=\sum_{i=2}^{n}\left[\begin{array}{c}
x_{i}^{C} \\
y_{i}^{C}
\end{array}\right]+\left[\begin{array}{c}
0 \\
\frac{h_{n}}{2}
\end{array}\right] .
$$

In case survey coordinate system has been implemented in WebGL environment, then it will be used to calculate new survey coordinates of the model and its objects in addition to the scene coordinates. Implementation and conversion of the coordinate systems is a complicated process presented in GIS environment that needs to be developed separately from the actual BIM model processing.

\section{Step 5. The rebuilding of the BIM model based on new coordinates.}

At the last step, the BIM model needs to be recompiled to use new scene coordinates that have dependencies with correlated coordinates. This step removes all old dependencies and coordinates in the model, adds defined rules and related objects parameters and assigns correlated coordinates to every object. The advantage of a consistent scene coordinate in the WebGL environment makes it possible to visualize the model correctly. Due to correlated coordinates, implemented rules and object's parameters, the analysis can be performed, for example, clash detection can be implemented in object movement function as a default in-object rules check.

\section{Conclusions}

The proposed approach extends opportunities of BIM model processing in the Web environment and allows implementation of the bigger amount of analysis that is based on the object's positioning and parameters. Due to implemented relations and rules, the objects in the BIM model can be considered not just like independent 3D objects in the WebGL environment as all object are presented by default, but as objects that are linked between each other and can act by the rules that defined to each type of the object. These rules are the main key factor that makes the difference between the usual 3D object and BIM model representation in the WebGL environment. Implemented correlated coordinates will be useful for the architects, building operators and facility managers who use the BIM model for the management of the building and its components. This method can be used to increase the effectiveness of space management, clash detection, relocation management and space programming.

The modelling of the existing conditions and lighting analysis can be done in a way that is more efficient due to the possibility of implementing the survey coordinate system that will assign the coordinates to each object in the scene. 
Unfortunately, the proposed approach of object's manipulation based on the objects' sequence has a disadvantage in comparison to the default manipulation methodology, and that is low performance. By the default, movement of the model is done as a group of objects, and that means that translate operation needs to be calculated just once, opposite to that in proposed. In the proposed method, the movement operation needs to be calculated for every object separately that leads to the bigger amount of operations and lower performance. This situation can be improved in further developments of this method by the implementation of the post-manipulation calculations and calculations on the request.

\section{References}

Borrmann, A., Kolbe, T. H., Donaubauer, A., Steuer, H., Jubierre, J. R., \& Flurl, M. (2015). Multi-scale geometric-semantic modeling of shield tunnels for GIS and BIM applications. Computer-Aided Civil and Infrastructure Engineering, 30(4), $263-281$. https://doi.org/10.1111/mice.12090

Dangermond, J. (2010). Foreword. In S. Rich, \& K. H. Davis (Eds.), Geographic Information Systems (GIS) for Facility Management. IFMA Foundation, USA.

Hor, A. H., Jadidi, A., \& Sohn, G. (2016). BIM-GIS integrated geospatial information model using semantic Web and RDF graphs. In ISPRS Annals of Photogrammetry, Remote Sensing \& Spatial Information Sciences, XXIII ISPRS Congress (vol. III-4), 12-19 July 2016, Prague, Czech Republic. https://doi.org/10.5194/isprsannals-III-4-73-2016

Hor, A.-H. (2015). A semantic Web platform for BIM-GIS integration (Technical Report). York University, Canada.

Irizarry, J., Karan, E. P., \& Jalaei, F. (2013). Integrating BIM and GIS to improve the visual monitoring of construction supply chain management. Automation in Construction, 31, 241-254. https://doi.org/10.1016/j.autcon.2012.12.005

Kang, T. W., \& Hong, C. H. (2015). A study on software architecture for effective BIM/GIS-based facility management data integration. Automation in Construction, 54, 25-38. https://doi.org/10.1016/j.autcon.2015.03.019

Karan, E. P., \& Irizarry, J. (2015). Extending BIM interoperability to preconstruction operations using geospatial analyses and semantic web services. Automation in Construction, 53, 1-12. https://doi.org/10.1016/j.autcon.2015.02.012

Kim, S. A., Shin, D., Choe, Y., Seibert, T., \& Walz, S. P. (2012). Integrated energy monitoring and visualization system for Smart Green City development: Designing a spatial information integrated energy monitoring model in the context of massive data management on a web based platform. Automation in Construction, 22, 51-59. https://doi.org/10.1016/j.autcon.2011.07.004

Li, Y., \& He, Z. (2008). 3D indoor navigation: a framework of combining BIM with 3D GIS. In 44 ${ }^{\text {th }}$ ISOCARP Congress (pp. 1$10)$.

Liu, X., Wang, X., Wright, G., Cheng, J., Li, X., \& Liu, R. (2017). A state-of-the-art review on the integration of Building Information Modeling (BIM) and Geographic Information System (GIS). ISPRS International Journal of Geo-Information, 6(2), 53. https://doi.org/10.3390/ijgi6020053

Ma, Z., \& Ren, Y. (2017). Integrated application of BIM and GIS: An overview. Procedia Engineering, 196, $1072-1079$. https://doi.org/10.1016/j.proeng.2017.08.064

Niu, S., Pan, W., \& Zhao, Y. (2015). A BIM-GIS integrated web-based visualization system for low energy building design. Procedia Engineering, 121, 2184-2192. https://doi.org/10.1016/j.proeng.2015.09.091

Pärn, E. A., Edwards, D. J., \& Sing, M. C. P. (2017). The building information modelling trajectory in facilities management: A review. Automation in Construction, 75, 45-55. https://doi.org/10.1016/j.autcon.2016.12.003

Pauwels, P., Zhang, S., \& Lee, Y. C. (2017). Semantic web technologies in AEC industry: A literature overview. Automation in Construction, 73, 145-165. https://doi.org/10.1016/j.autcon.2016.10.003

Wong, J. K. W., Ge, J., \& He, S. X. (2018). Digitisation in facilities management: A literature review and future research directions. Automation in Construction, 92, 312-326. https://doi.org/10.1016/j.autcon.2018.04.006

Zhu, J., Wright, G., Wang, J., \& Wang, X. (2018). A critical review of the integration of geographic information system and building information modelling at the data level. ISPRS International Journal of Geo-Information, 7(2), 66. https://doi.org/10.3390/ijgi7020066 